\title{
Automatic MRI Quantifying Methods in Behavioral-Variant Frontotemporal Dementia Diagnosis
}

\author{
Antti Cajanus $^{a}{ }^{b}$ Anette Hall ${ }^{a}$ Juha Koikkalainen ${ }^{c}$ Eino Solje ${ }^{a, b}$ \\ Antti Tolonen $^{d}$ Timo Urhemaa $^{d}$ Yawu Liu ${ }^{a, e}$ Ramona M. Haanpääa \\ Päivi Hartikainen ${ }^{a, b}$ Seppo Helisalmi ${ }^{a}$ Ville Korhonen ${ }^{a, f}$ \\ Daniel Rueckert ${ }^{9}$ Steen Hasselbalch ${ }^{h}$ Gunhild Waldemar ${ }^{h}$ \\ Patrizia Mecocci ${ }^{i}$ Ritva Vanninen ${ }^{\text {e }}$ Mark van Gils $^{d}$ Hilkka Soininen ${ }^{a}$ b \\ Jyrki Lötjönen ${ }^{c} \quad$ Anne M. Remes ${ }^{a, b}, j, k$

\begin{abstract}
${ }^{a}$ Institute of Clinical Medicine - Neurology, University of Eastern Finland, Kuopio, Finland;
${ }^{b}$ Neurocenter, Neurology, Kuopio University Hospital, Kuopio, Finland; ' ${ }^{~ C o m b i n o s t i c s ~ L t d ., ~}$ Tampere, Finland; dVTT Technical Research Centre of Finland Ltd., Tampere, Finland; e Department of Radiology, Kuopio University Hospital, Kuopio, Finland; ${ }^{f}$ Neurocenter, Neurosurgery, Kuopio University Hospital, Kuopio, Finland; 9 Department of Computing, Imperial College London, London, UK; h Danish Dementia Research Centre, Department of Neurology, Rigshospitalet, University of Copenhagen, Copenhagen, Denmark; 'Section of Gerontology and Geriatrics, University of Perugia, Perugia, Italy; ${ }^{j}$ Medical Research Center, Oulu University Hospital, Oulu, Finland; ' Unit of Clinical Neuroscience, Neurology, University of Oulu, Oulu, Finland
\end{abstract}

\section{Keywords}

Frontotemporal dementia $\cdot$ Frontotemporal lobar degeneration · Neuroimaging $\cdot$ MRI .

Dementia $\cdot$ Machine learning

\begin{abstract}
Aims: We assessed the value of automated MRI quantification methods in the differential diagnosis of behavioral-variant frontotemporal dementia (bvFTD) from Alzheimer disease (AD), Lewy body dementia (LBD), and subjective memory complaints (SMC). We also examined the role of the C9ORF72-related genetic status in the differentiation sensitivity. Methods: The MRI scans of 50 patients with bvFTD (17 C9ORF72 expansion carriers) were analyzed using 6 quantification methods as follows: voxel-based morphometry (VBM), tensor-based morphometry, volumetry (VOL), manifold learning, grading, and white-matter hyperintensities. Each patient was then individually compared to an independent reference group in order to attain diagnostic suggestions. Results: Only VBM and VOL showed utility in correctly identifying bvFTD from our set of data. The overall classification sensitivity of bvFTD with VOL + VBM achieved a total sensitivity of $60 \%$. Using VOL + VBM, $32 \%$ were misclassified as having LBD. There was a trend of higher values for classification sensitivity of the C9ORF72 expansion carriers than
\end{abstract}


noncarriers. Conclusion: VOL, VBM, and their combination are effective in differential diagnostics between bvFTD and AD or SMC. However, MRI atrophy profiles for bvFTD and LBD are too similar for a reliable differentiation with the quantification methods tested in this study.

(C) 2018 The Author(s)

Published by S. Karger AG, Basel

\section{Introduction}

Frontotemporal lobar degeneration (FTLD) is the second most common early-onset dementing disease. FTLD is a heterogeneous group of different syndromes and also patterns of brain atrophy that vary between different phenotypes. A behavioral-variant frontotemporal dementia (bvFTD) is the most common phenotype of FTLD. Nearly one-half of the identified cases are of familial origin. While the genetic etiology of bvFTD is variable, the C9ORF72 expansion represents the most common genetic etiology, which is considered to underlie as many as over $30 \%$ of familial bvFTD cases $[1,2]$.

The accurate differential diagnostics of neurodegenerative diseases is crucial, not only from the perspective of clinical work, but also for the development of new treatments as well as the identification of patients suitable for clinical trials. The clinical diagnosis of bvFTD during a patient's lifetime is based on a diagnostic battery defined by the international bvFTD criteria consortium, including clinical symptoms, neuropsychological assessment for possible bvFTD, and in addition imaging and evaluation of functional decline for probable bvFTD. The definite diagnosis would require pathological confirmation or genetic testing, which are seldom available [3, 4]. However, the diagnostics is challenging and the sensitivity of the criteria for possible bvFTD varies between 85 and $95 \%$ and for probable bvFTD between 75 and $85 \%$ in different studies $[3,5,6]$. On the other hand, the specificity of the criteria for possible bvFTD varies between 27 and $82 \%$ and for probable bvFTD between 85 and 95\%, emphasizing the value of imaging in the differential diagnosis from other neurodegenerative disorders. However, visual evaluation of magnetic resonance imaging (MRI) requires an experienced neuroradiologist, and even so, the visual assessment provides only $59 \%$ sensitivity and $80 \%$ specificity from other common dementing diseases and is vulnerable for inter-rater differences [7]. It has also been proposed that different genetic forms of bvFTD are associated with different types of brain atrophy. In addition to frontal and temporal atrophy, it has been suggested that the carriers of the C9ORF72 expansion exhibit more widespread atrophy affecting the occipital lobes, cerebellum, and subcortical grey matter [8-12].

In the future, automatic decision support tools may be helpful as a part of differential diagnostics of neurodegenerative disorders. The Disease State Index (DSI) is a statistical classifier developed for clinical work as a decision support tool. It is designed to utilize multimodal data for analysis to provide a score for individual patient classification. This method has previously shown to be useful in diagnosing Alzheimer disease (AD), predicting the progression of patients with mild cognitive impairment into AD, differentiating FTLD from $\mathrm{AD}$ and mild cognitive impairment using multimodal data [13-16]. Its performance between different patient cohorts has also been validated [17].

The aim of this study was to evaluate the utility of the DSI using data from MRI scans only in a setting of differentiating bvFTD from AD, FTLD, Lewy body dementia (LBD) and subjective memory complaints (SMC). We performed DSI analysis individually for each patient with separate reference data, mimicking a clinical setting. We also examined if there was a difference in the sensitivity of the classification between the carriers and noncarriers of the C9ORF72 expansion. 
Cajanus et al.: Automatic MRI Quantifying Methods in Behavioral-Variant Frontotemporal Dementia Diagnosis

Table 1. Patient cohort characteristics: demographic and clinical data of both the $C 9 O R F 72$ expansion carriers and noncarriers

\begin{tabular}{lrrrr}
\hline & C9ORF72+ & C9ORF72- & Total & $p$ \\
\hline Subjects, $n(\%)$ & $17(34)$ & $33(66)$ & $50(100)$ & 0.4 \\
Female gender, $n(\%)$ & $9(53)$ & $16(49)$ & $25(50)$ & 0.8 \\
Mean age at scan \pm SD, years & $58.6 \pm 8.2$ & $64.5 \pm 7.4$ & $62.5 \pm 8.1$ & 0.02 \\
Mean MMSE score \pm SD & $21.5 \pm 6.3$ & $23.2 \pm 4.1$ & $22.6 \pm 4.9$ & 0.3 \\
Mean time from symptoms to MRI \pm SD, years & $3.1 \pm 3.1$ & $2.4 \pm 3.1$ & $2.6 \pm 3.1$ & 0.5 \\
\hline
\end{tabular}

Age at scan was significantly lower in the C9ORF72 expansion carrier group. No other statistically significant differences emerged. MMSE, Mini-Mental State Examination.

\section{Materials and Methods}

\section{Subjects}

A total of 50 bvFTD patients diagnosed with current diagnostic guidelines were included in this study (Table 1) [3]. The cohort included men and women equally and the mean age was 62.5 years (36-79). Comorbidity with amyotrophic lateral sclerosis (bvFTD-ALS) was diagnosed in $10 \%(n=5)$ of patients. The C9ORF72 expansion was detected in $34 \%(n=17)$ of patients, while the remaining $66 \%(n=33)$ were negative for the mutation. Analyses of the demographic data indicated that at the time of the MRI scan, the patients in the C9ORF72 expansion carrier group as compared to those with a negative status were significantly younger (mean age 60.5 vs. 65.5 years, respectively, $p=0.02$ ). Otherwise there was no difference between the C9ORF72 expansion carriers and noncarriers.

SMC subjects have entered a memory clinic with subjective memory problems, but without any objective finding of a neurodegenerative process. We included these patients as a reference to evaluate if the MRI quantification methods are able to determine patients with and without neurodegenerative process.

All patients were diagnosed by an experienced neurologist specialized in memory disorders in the University Hospital of Kuopio, Finland. The ethics committee of the University Hospital of Kuopio approved the research protocol in accordance with the principles of the Declaration of Helsinki. Each patient provided written informed consent to participate in the study.

\section{Genetic Analysis}

Genomic DNA was extracted from venous blood samples using QIAamp DNA blood mini extraction kit (Qiagen). The presence of the C9ORF72 expansion was detected using repeatprimed PCR [2]. Of those testing positive for the expansion, 18 had an excess of 40 repeats, with 3 cases showing an intermediate expansion length between 10 and 40 repeats. Patients were considered negative for the C9ORF72 expansion if their number of repeats was fewer than 10 [2].

\section{MRI Acquisition}

For all patients, brain MRI was performed using either a 1.5-T or a 3-T MRI scanner: $56 \%$ $(n=28)$ of cases were scanned with a 1.5-T scanner, while the remaining $44 \%(n=22)$ patients underwent a 3-T scanner. In 49 patients, both a T1-weighted 3D gradient echo sequence and a fast fluid-attenuated inversion recovery (FLAIR) sequence were obtained in MRI. The FLAIR sequence was unavailable for 1 patient. In a previous study with multiple cohorts, no notable effects of magnetic field strength between $1.5 \mathrm{~T}$ and $3 \mathrm{~T}$ for imaging biomarkers were found [18]. 
Cajanus et al.: Automatic MRI Quantifying Methods in Behavioral-Variant

Frontotemporal Dementia Diagnosis

\section{Image Analysis}

The MRI analysis was performed with 6 fully automatic image analysis methods quantifying the following features: volume of various brain areas (VOL), local volume change using tensor-based morphometry (TBM), local grey matter changes using voxel-based morphometry (VBM), image similarity to images in the reference database using manifold-based learning (Manifold) and grading, as well as white-matter hyperintensities (WMH). All imaging biomarkers were generated using solely T1 and FLAIR images. We did the normalization by gender and age using the SMC patients as the reference group for VOL, VBM, TBM, and WMH biomarker values. We fitted a linear regression model using age and gender as explanatory variables for the MRI values, which were then transformed to match the age and gender of the patient being analyzed using these regression models [19]. We analyzed patients using each quantification method individually and all possible combinations of methods, in order to evaluate the diagnostic value of each method, which has been previously described [18].

The MRI scans of the 50 bvFTD patients were performed in Kuopio University Hospital to support the patients' original diagnosis. The scans were originally evaluated by a radiologist specialized in neurodegenerative disorders as part of the initial diagnosing process. For this study, the scans were retrospectively collected from the patient data archive. The radiologist's expert opinion was not used in this study, only the parameters extracted by the automatic MRI quantification methods were used to classify patients into predetermined diagnostic categories (AD, FTLD, LBD, and SMC).

\section{Reference Data}

The reference data included a total of 1,111 patients who were evaluated in 4 different centers (VU Medical Center, The Netherlands; Università degli Studi di Perugia, Italy; Rigshospitalet, Denmark; and University of Eastern Finland, Finland). Additionally, data from the Alzheimer's Disease Neuroimaging Initiative (ADNI) were included. The reference data were completely independent of the study group. The reference data included patients pertaining to 4 major diagnostic groups as follows: AD $(n=537)$, FTLD $(n=154)$, LBD $(n=61)$, and SMC $(n=$ 359). In the reference data, the FTLD group included patients with bvFTD, non-fluent-variant primary progressive aphasia, and semantic-variant primary progressive aphasia, thus we refer to the group as FTLD, not bvFTD. The reference data are described in online supplementary Table 1 (see www.karger.com/doi/10.1159/000486849 for all online suppl. material) [20].

\section{Statistical Analysis}

Both the demographic and the clinical data were compared between groups of the C9ORF72 expansion carriers and noncarriers by applying Student $t$ tests and Pearson $\chi^{2}$ tests. The Pearson $\chi^{2}$ test was performed in order to explore differences in the classification sensitivity between the genetic groups. $p$ values $<0.05$ were considered as statistically significant. The statistical analyses were performed using the IBM ${ }^{\circledR}$ SPSS ${ }^{\circledR}$ Statistics software package, version 22.

The decision support tool used in this study, the DSI, generates a numerical measure for each patient, in order to evaluate the state of the disease progression on a scale of $0-1$. The DSI is designed to use multimodal data in the differentiating process, but in this study we investigated the utility of features extracted only from MRI scans. By combining all available biomarkers extracted from the MRI scans with the quantification methods specified earlier, the DSI generates an individual patient's profile. The DSI measures the fit of the patient data with reference to previously diagnosed cases from two groups. More specifically, a similarity to the reference group leads to a low DSI value, while a similarity to the study group results in a high DSI value. This pairwise comparison between two diagnostic categories is repeated for each possible pair, and finally the classifier suggests the most likely diagnosis for each 


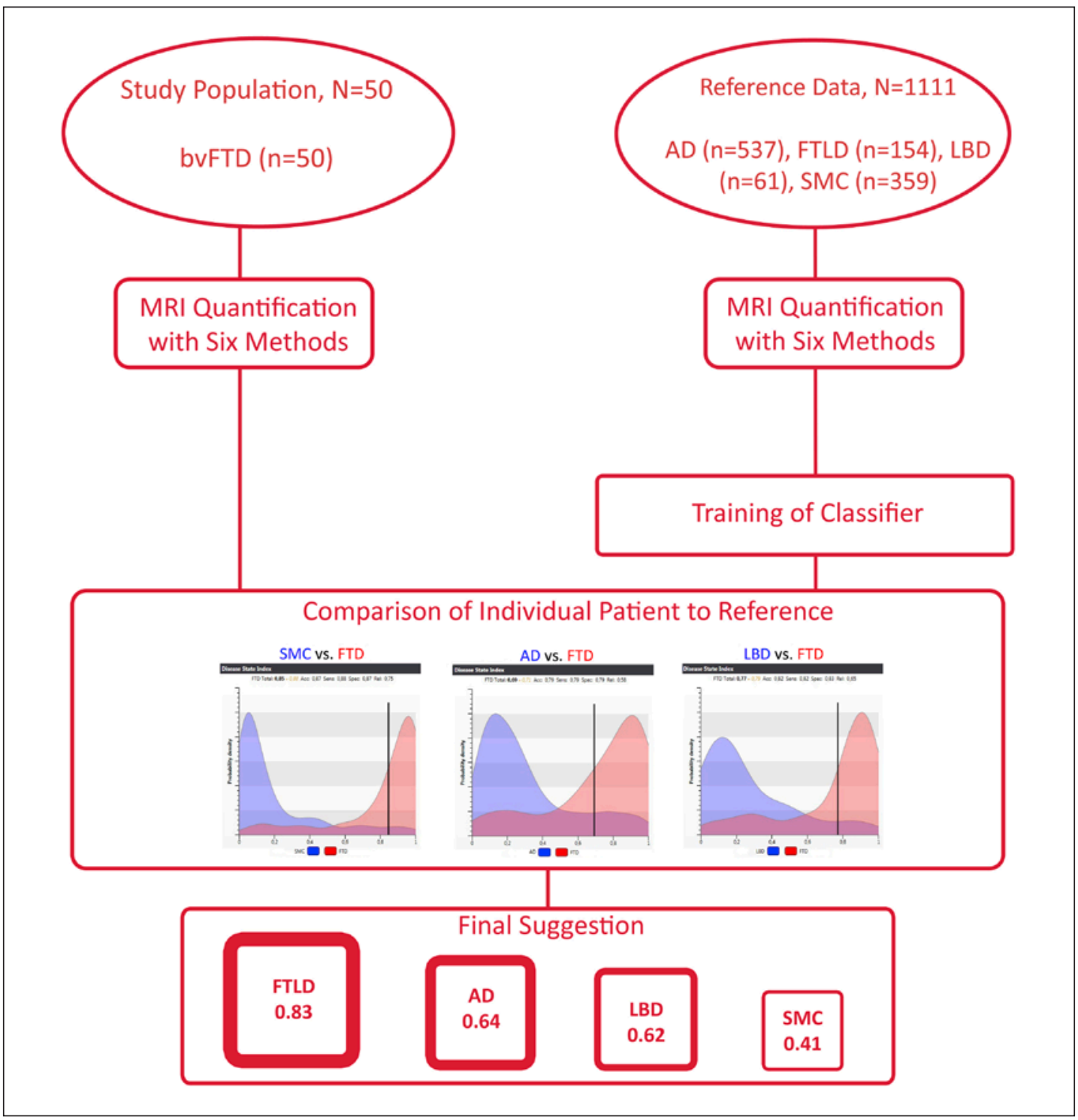

Fig. 1. Illustration of the study setting. Our study population comprised 50 bvFTD patients. They all were analyzed with 6 MRI quantification methods. After the analysis, each patient was individually compared to reference data of previously diagnosed cases. Finally, the DSI suggested the most likely diagnosis based on the resemblance of the patient's and reference MRI scans.

individual patient based on multiple volumetric and morphometric measures normalized by sex and age $[14,18]$. In this study, 4 diagnostic classes were used: FTLD, LBD, AD, and SMC. An illustration of the study setting is presented in Figure 1.

\section{Results}

The DSI performed the best using only MRI parameters extracted with VBM in the classification process. In total bvFTD cohort, the DSI achieved sensitivity (56\%) by using only VBM of the 6 individual quantification methods. Using VBM, the sensitivity was even higher 
Table 2. Classification of the 50 bvFTD cases into diagnostic categories with different MRI classification methods

\begin{tabular}{l|l}
\hline Dement Geriatr Cogn Disord Extra 2018;8:51-59 \\
\hline DOI: 10.1159/000486849 & $\begin{array}{l}\text { ( ) 2018 The Author(s). Published by S. Karger AG, Basel } \\
\text { www.karger.com/dee }\end{array}$ \\
\hline
\end{tabular}

Cajanus et al.: Automatic MRI Quantifying Methods in Behavioral-Variant Frontotemporal Dementia Diagnosis

\begin{tabular}{lcrlc}
\hline Methods used & \multicolumn{1}{l}{ FTLD } & \multicolumn{1}{c}{ AD } & \multicolumn{1}{l}{ LBD } & \multicolumn{1}{l}{ SMC } \\
\hline VOL & $22(44)$ & $8(16)$ & $14(28)$ & $6(12)$ \\
TBM & $11(22)$ & $18(36)$ & $17(34)$ & $4(8)$ \\
VBM & $28(56)$ & $7(14)$ & $14(28)$ & $1(2)$ \\
WMH & $9(18)$ & $22(44)$ & $4(8)$ & $15(30)$ \\
Grading & $13(26)$ & $5(10)$ & $16(32)$ & $16(32)$ \\
Manifold & $4(8)$ & $15(30)$ & $26(52)$ & $5(10)$ \\
VOL + VBM & $30(60)$ & $2(4)$ & $16(32)$ & $2(4)$ \\
\hline
\end{tabular}

Values are shown as $n(\%)$. bvFTD, behavioral-variant frontotemporal dementia; FTLD, frontotemporal lobar degeneration; AD, Alzheimer disease; LBD, Lewy body dementia; SMC, subjective memory complaints; VOL, volumetry; TBM, tensor-based morphometry; VBM, voxel-based morphometry; WMH, white-matter hyperintensities; manifold, manifold-based learning.
Table 3. Classification of the C9ORF72 expansion carriers/ noncarriers into diagnostic categories with the best performing MRI classification methods

\begin{tabular}{lrlrl}
\hline & FTLD & AD & LBD & SMC \\
\hline $\begin{array}{l}\text { VOL + VBM } \\
\text { C9ORF72+ }\end{array}$ & $11(65)$ & $1(6)$ & $5(29)$ & 0 \\
$\begin{array}{l}\text { C9ORF72- } \\
\text { VOL }\end{array}$ & $19(58)$ & $1(3)$ & $11(33)$ & $2(6)$ \\
C9ORF72+ & $8(47)$ & $3(18)$ & $5(29)$ & $1(6)$ \\
C9ORF72- & $14(42)$ & $5(15)$ & $9(27)$ & $5(15)$ \\
VBM & $12(71)$ & $2(12)$ & $3(18)$ & 0 \\
C9ORF72+ & $16(48)$ & $4(12)$ & $12(36)$ & $1(3)$ \\
C9ORF72- & 16 & & \\
\hline
\end{tabular}

Values are shown as $n(\%)$. Suggestions for diagnostic categories as per quantification methods. The rows represent the methods used and the genetic subgroups of the C9ORF72 expansion carriers and noncarriers, while the columns represent the diagnostic suggestion. No statistically significant differences in differentiation accuracy between the genetic groups were found using only VOL and/or VBM. FTLD, frontotemporal lobar degeneration; AD, Alzheimer disease; LBD, Lewy body dementia; SMC, subjective memory complaints; VOL, volumetry; VBM, voxel-based morphometry.

in patients with the C9ORF72 expansion (71\%) (Table 3). Sensitivity of $44 \%$ was detected in the total group of bvFTD by using VOL. The other 4 methods (TBM, grading, WMH, and manifold) showed no utility in classification. Overall, there was a trend of higher values for classification sensitivity of the C9ORF72 expansion carriers than noncarriers. Classification sensitivities of all quantification methods are presented in Table 2.

The best combination of quantification methods was found to be VBM + VOL giving a classification sensitivity of $60 \%$ for the whole bvFTD cohort. By using the combination of VBM and VOL, the carriers of the C9ORF72 expansion were classified with $65 \%$ and noncarriers with 58\% sensitivity (Table 3). However, this difference did not reach statistical significance ( $p=0.37$ ). Classification sensitivities of all possible combinations of quantification methods are presented in online supplementary Table 2. 
Cajanus et al.: Automatic MRI Quantifying Methods in Behavioral-Variant

Frontotemporal Dementia Diagnosis

With the combination of VOL + VBM, patients were most often misclassified as having LBD (32\%), while $4 \%$ were misclassified as AD patients, and the remaining $4 \%$ as SMC subjects.

\section{Discussion}

The aim of the current study was to evaluate the utility of 6 automated MRI quantification methods specifically in performing differential diagnostics of bvFTD. We found that only a small subset of imaging biomarkers were useful in the classification of bvFTD. More specifically, only VBM and VOL together with grading reached a sensitivity greater than $25 \%$, which represents the sensitivity we would achieve by complete chance in this 4-class-scenario. VBM was the best method giving a sensitivity of $56 \%$ in the whole bvFTD group. However, the sensitivity was even higher (71\%) in patients with the C9ORF72 expansion. The combination of VBM + VOL improved the classification sensitivity with the C9ORF72 expansion noncarriers, while with the $C 90 R F 72$ expansion carriers the sensitivity was slightly reduced.

In a previous study utilizing identical MRI analysis methods, there was a differentiation accuracy of $62 \%$ of FTLD patients from SMC, AD, LBD, and vascular dementia patients [18]. In that study, $21 \%$ of FTLD patients were misclassified as having AD, which is more than in the present study (4\%). However, in the previous study, only 5\% of FTLD patients were misclassified as having LBD, which contrasts strongly with the respective value in the present study. One reason for this may be that the patient cohort differed in clinical characteristics from our cohort. In the current study, even the best performing quantification method VBM resulted in $28 \%$ of the patients being misclassified as having LBD. Among the C9ORF72 expansion carriers, the classification was better: only $18 \%$ of the carriers and $36 \%$ of the noncarriers were incorrectly classified as having LBD with VBM. Potential explanations for this result are as follows: first, at least some of the C9ORF72 expansion carriers may have been associated with overlapping neurodegenerative conditions in addition to bvFTD. Second, as the diagnosis of the C9ORF72 expansion noncarriers was solely based on the clinical evaluation of symptoms due to objective biomarkers lacking (the clinical diagnosis is considered to reach $85-95 \%$ specificity) $[3,5,6]$, this patient group may have included some individuals with a clinical misdiagnosis of bvFTD. Thirdly, LBD is associated with widespread cortical and central atrophy with no specific MRI findings [21-23]. Existing evidence suggests that specifically in the C9ORF72 expansion carrier bvFTD patients, the atrophy may affect the posterior regions of the brain as well as the frontal and temporal regions $[11,12,24]$. The genetic status of the reference FTLD cases is not known and the dataset may include a number of cases with the C9ORF72 expansion. The reference data also includes other clinical phenotypes of FTLD, such as progressive aphasia and semantic dementia, in addition to bvFTD. Moreover, the confusion between LBD and bvFTD may also be associated with a long disease progression of bvFTD prior to the MRI scan, leading to a more general and widespread atrophy, which would make MRI biomarkers for bvFTD and LBD relatively similar to each other. The relatively low sample size of the study may emphasize the influence of chance and errors in the clinical diagnosis. No patients with mild cognitive impairment were included in the reference data, since the underlying neurodegenerative process at that stage is uncertain. Defining the ground truth diagnosis for those patients would most likely produce a significant amount of false diagnoses.

It is important to note here that in a multi-class setting, the classification accuracies are much lower than those associated with pairwise comparison settings, thus rendering findings between such studies not strictly comparable with each other $[25,26]$. In light of the current study, it is important to note that the multi-class setting provided herein is associated with a 
Cajanus et al.: Automatic MRI Quantifying Methods in Behavioral-Variant

Frontotemporal Dementia Diagnosis

higher ecological validity than that of a pairwise setting, bearing a closer resemblance to the real-life scenario faced by a patient suffering from a neurodegenerative disorder and undergoing a differential diagnostic procedure. However, the validation of different methods and diagnostic tools in several cohorts is needed to provide high quality tools.

\section{Conclusion}

We demonstrated high differentiation sensitivity of bvFTD from the most common dementing neurodegenerative disorder (AD) by using MRI quantification applications. Similarly, SMC subjects were differentiated from bvFTD with high sensitivity. However, out of the 6 image quantification methods assessed, only 2 methods, VBM and VOL, were found to be useful in the differentiation of bvFTD from other disorders. The best classification sensitivity was achieved by using the VBM method. This clearly implicates that further studies are needed to both evaluate the feasibility of various MRI quantifying methods and to optimize the set of features that are useful in the differential diagnosis of neurodegenerative dementing diseases. The most challenging differentiation was found between bvFTD and LBD, due to similarities in the MRI profiles characterizing both groups of patients. Automated MRI quantification methods and decision support tools may be helpful in the differential diagnostics of bvFTD in clinical practice. However, in the case of the more uncommon neurodegenerative disorders, such as bvFTD and LBD, differential diagnostics using only MRI methods seems to be challenging and also the diagnosis of specific clinical features and other biomarkers should be included in decision support tools to provide greater sensitivity and specificity.

\section{Acknowledgements}

This work received funding from the following bodies: European Union's Seventh Framework Programme for research, technological development, and demonstration under grant agreement No. 611005 (PredictND); VTR funding from Kuopio University Hospital; the Finnish Medical Foundation; the Olvi Foundation; the Finnish Alzheimer Research Association; the Finnish Brain Foundation; and the Päivikki and Sakari Sohlberg foundation.

\section{Disclosure Statement}

Equity: Juha Koikkalainen and Jyrki Lötjönen are shareholders and founders of Combinostics Ltd. Patents/royalties: Combinostics Ltd. owns the following IPR related to the paper: 1. J. Koikkalainen and J. Lötjönen. A method for inferring the state of a system, US7,840,510 B2, PCT/FI2007/050277. 2. J. Lötjönen, J. Koikkalainen, and J. Mattila. State inference in a heterogeneous system, PCT/FI2010/050545, FI20125177. The other authors have no conflicts of interest to disclose.

\section{References}

1 Majounie E, Renton AE, Mok K, Dopper EGP, Waite A, Rollinson S, et al: Frequency of the C90RF72 hexanucleotide repeat expansion in patients with amyotrophic lateral sclerosis and frontotemporal dementia: a crosssectional study. Lancet Neurol 2012;11:323-330.

$\longrightarrow 2$ Renton AE, Majounie E, Waite A, Simón-Sánchez J, Rollinson S, Gibbs JR, et al: A hexanucleotide repeat expansion in C90RF72 is the cause of chromosome 9p21-linked ALS-FTD. Neuron 2011;72:257-268. 
3 Rascovsky K, Hodges JR, Knopman D, Mendez MF, Kramer JH, Neuhaus J, et al: Sensitivity of revised diagnostic criteria for the behavioural variant of frontotemporal dementia. Brain 2011;134:2456-2477.

4 Gorno-Tempini ML, Hillis AE, Weintraub S, Kertesz A, Mendez M, Cappa SF, et al: Classification of primary progressive aphasia and its variants. Neurology 2011;76:1006-1014.

5 Harris JM, Gall C, Thompson JC, Richardson AMT, Neary D, du Plessis D, et al: Sensitivity and specificity of FTDC criteria for behavioral variant frontotemporal dementia. Neurology 2013;80:1881-1887.

-6 Balasa M, Gelpi E, Martín I, Antonell A, Rey MJ, Grau-Rivera O, et al: Diagnostic accuracy of behavioral variant frontotemporal dementia consortium criteria (FTDC) in a clinicopathological cohort. Neuropathol Appl Neurobiol 2015;41:882-892.

7 Harper L, Fumagalli GG, Barkhof F, Scheltens P, O’Brien JT, Bouwman F, et al: MRI visual rating scales in the diagnosis of dementia: evaluation in 184 post-mortem confirmed cases. Brain 2016;139:1211-1225.

8 Mahoney CJ, Downey LE, Ridgway GR, Beck J, Clegg S, Blair M, et al: Longitudinal neuroimaging and neuropsychological profiles of frontotemporal dementia with C90RF72 expansions. Alzheimers Res Ther 2012;4:41.

-9 Mahoney CJ, Beck J, Rohrer JD, Lashley T, Mok K, Shakespeare T, et al: Frontotemporal dementia with the C90RF72 hexanucleotide repeat expansion: clinical, neuroanatomical and neuropathological features. Brain 2012;135:736-750.

10 Sha SJ, Takada LT, Rankin KP, Yokoyama JS, Rutherford NJ, Fong JC, et al: Frontotemporal dementia due to C90RF72 mutations: clinical and imaging features. Neurology 2012;79:1002-1011.

11 Whitwell JL, Weigand SD, Boeve BF, Senjem ML, Gunter JL, Dejesus-Hernandez M, et al: Neuroimaging signatures of frontotemporal dementia genetics: C9ORF72, tau, progranulin and sporadics. Brain 2012;135:794806.

12 Bocchetta M, Cardoso MJ, Cash DM, Ourselin S, Warren JD, Rohrer JD: Patterns of regional cerebellar atrophy in genetic frontotemporal dementia. NeuroImage Clin 2016;11:287-290.

-13 Hall A, Mattila J, Koikkalainen J, Lötjonen J, Wolz R, Scheltens P, et al: Predicting progression from cognitive impairment to Alzheimer's disease with the Disease State Index. Curr Alzheimer Res 2015;12:69-79.

14 Mattila J, Koikkalainen J, Virkki A, Simonsen A, Van Gils M, Waldemar G, et al: A disease state fingerprint for evaluation of Alzheimer's disease. J Alzheimers Dis 2011;27:163-176.

15 Mattila J, Soininen H, Koikkalainen J, Rueckert D, Wolz R, Waldemar G, et al: Optimizing the diagnosis of early Alzheimer's disease in mild cognitive impairment subjects. J Alzheimers Dis 2012;32:969-979.

-16 Muñoz-Ruiz MÁ, Hartikainen P, Hall A, Mattila J, Koikkalainen J, Herukka S-K, et al: Disease state fingerprint in frontotemporal degeneration with reference to Alzheimer's disease and mild cognitive impairment. J Alzheimers Dis 2013;35:727-739.

17 Hall A, Muñoz-Ruiz M, Mattila J, Koikkalainen J, Tsolaki M, Mecocci P, et al: Generalizability of the disease state index prediction model for identifying patients progressing from mild cognitive impairment to Alzheimer's disease. J Alzheimers Dis 2015;44:79-92.

18 Koikkalainen J, Rhodius-Meester H, Tolonen A, Barkhof F, Tijms B, Lemstra AW, et al: Differential diagnosis of neurodegenerative diseases using structural MRI data. NeuroImage Clin 2016;11:435-449.

19 Koikkalainen J, Pölönen H, Mattila J, van Gils M, Soininen H, Lötjönen J: Improved classification of Alzheimer's disease data via removal of nuisance variability. PLoS One 2012;7:e31112.

-20 Van Der Flier WM, Pijnenburg YAL, Prins N, Lemstra AW, Bouwman FH, Teunissen CE, et al: Optimizing patient care and research: the Amsterdam dementia cohort. J Alzheimers Dis 2014;41:313-327.

21 O'Brien JT, Paling S, Barber R, Williams ED, Ballard C, McKeith IG, et al: Progressive brain atrophy on serial MRI in dementia with Lewy bodies, AD, and vascular dementia. Neurology 2001;56:1386-1388.

22 Whitwell JL, Jack CR, Parisi JE, Knopman DS, Boeve BF, Petersen RC, et al: Rates of cerebral atrophy differ in different degenerative pathologies. Brain 2007;130:1148-1158.

-23 Nedelska Z, Ferman TJ, Boeve BF, Przybelski SA, Lesnick TG, Murray ME, et al: Pattern of brain atrophy rates in autopsy-confirmed dementia with Lewy bodies. Neurobiol Aging 2015;36:452-461.

24 Kaivorinne A-L, Bode MK, Paavola L, Tuominen H, Kallio M, Renton AE, et al: Clinical Characteristics of C90RF72-linked frontotemporal lobar degeneration. Dement Geriatr Cogn Dis Extra 2013;3:251-262.

-25 Canu E, Agosta F, Mandic-Stojmenovic G, Stojković T, Stefanova E, Inuggi A, et al: Multiparametric MRI to distinguish early onset Alzheimer's disease and behavioural variant of frontotemporal dementia. NeuroImage Clin 2017;15:428-438.

-26 Meyer S, Mueller K, Stuke K, Bisenius S, Diehl-Schmid J, Jessen F, et al: Predicting behavioral variant frontotemporal dementia with pattern classification in multi-center structural MRI data. NeuroImage Clin 2017;14: 656-662. 